\title{
Avaliação da toxicidade das tinturas de aroeira e de romã através do bioensaio com
}

\section{Artemia salina}

\author{
Toxicity assessment of mastic and pomegranate tinctures using the Artemia salina bioassay \\ Evaluación de la toxicidad de las tinturas de masilla y granada mediante el bioensayo de Artemia \\ salina
}

Recebido: 10/03/2021 | Revisado: 17/03/2021 | Aceito: 17/03/2021 | Publicado: 24/03/2021

\author{
Ana Cristina Rodrigues Martins \\ ORCID: https://orcid.org/0000-0002-9082-0402 \\ Faculdade Paulo Picanço, Brasil \\ E-mail: aniniha_acrm@hotmail.com \\ Jane Kenya Nogueira da Costa \\ ORCID: https://orcid.org/0000-0003-0192-2560 \\ Faculdade Paulo Picanço, Brasil \\ E-mail: janekenya@gmail.com \\ Adyson Herbert \\ ORCID: https://orcid.org/0000-0003-2890-9534 \\ Faculdade Paulo Picanço, Brasil \\ E-mail: adyson.herbert@facpp.edu.br \\ Fabrício Rômulo Sampaio Farias \\ ORCID: https://orcid.org/0000-0002-6119-6890 \\ Faculdade Paulo Picanço, Brasil \\ E-mail: fabricio.farias@ facpp.edu.br \\ Márcia Rezende \\ ORCID: https://orcid.org/0000-0001-8474-5656 \\ Faculdade Paulo Picanço, Brasil \\ E-mail: marcia.rezende@ facpp.edu.br \\ Vitoldo Antonio Kozlowski Junior \\ ORCID: https://orcid.org/0000-0002-1339-4614 \\ Universidade Estadual de Ponta Grossa, Brasil \\ E-mail: vakozlowski@uepg.br \\ Juliana Larocca de Geus \\ ORCID: https://orcid.org/0000-0001-9633-0474 \\ Faculdade Paulo Picanço, Brasil \\ E-mail: juliana.degeus@facpp.edu.br
}

\begin{abstract}
Resumo
Este trabalho teve como objetivo avaliar a toxicidade das tinturas de aroeira (Myracrodruon urundeuva) e de romã (Punica granatum) por meio do bioensaio com Artemia salina. Diferentes concentrações das tinturas $(25,50,75,150$, 250, 500, 750 e $1000 \mu \mathrm{g} / \mathrm{mL}$ ) foram adicionadas aos náuplios e a concentração letal média (CL50) foi avaliada, utilizando a análise de Probit através do software StatPlus® (AnalystSoft, USA), obtendo-se os intervalos de confiança superior e inferior, após um período de exposição de 24 horas. Os extratos foram considerados ativos quando os valores foram < $1000 \mathrm{ug} / \mathrm{mL}$. Curvas de sobrevivência e testes de LogRank foram realizados para todos os extratos na concentração de $250 \mu \mathrm{g} / \mathrm{mL}$ contra controle negativo (Salina) e controle positivo (Clorexidina), utilizando o software GraphPad ${ }^{\circledR}$ Prism (GraphPad Software, USA). Para a aroeira a CL50 foi de 607,87 \pm 38,73, já para a romã, de 464,98 $\pm 28,72$, sendo ambas as tinturas consideradas ativas. Quando comparadas à solução salina, houve diferença significativa nas curvas de sobrevivência $(p=0,0206)$, o mesmo ocorreu para as comparações com a clorexidina $(\mathrm{p}<0,0001)$. Pode-se concluir que as tinturas de aroeira e de romã foram tóxicas contra a Artemia salina nas diferentes concentrações testadas.
\end{abstract}

Palavras-chave: Punicaceae; Bursera; Testes de Toxicidade.

\begin{abstract}
This work aimed to evaluate the toxicity of the mastic (Myracrodruon urundeuva) and pomegranate (Punica granatum) tinctures through the bioassay with Artemia salina. Different dye concentrations (25, 50, 75, 150, 250, 500, 750 and $1000 \mu \mathrm{g} / \mathrm{mL}$ ) were added to the nauplii and the average lethal concentration (CL50) was evaluated using Probit analysis using the StatPlus® software (AnalystSoft, USA), obtaining the upper and lower confidence intervals, after a 24-hour exposure period. The extracts were considered active when the values were $<1000 \mathrm{ug} / \mathrm{mL}$. Survival
\end{abstract}


curves and LogRank tests were performed for all extracts at a concentration of $250 \mu \mathrm{g} / \mathrm{mL}$ against negative control (Saline) and positive control (Chlorhexidine), using the GraphPad® Prism software (GraphPad Software, USA). For the mastic, the LC50 was $607.87 \pm 38.73$, for the pomegranate, $464.98 \pm 28.72$, both tinctures being considered active. When compared to saline, there was a significant difference in survival curves $(\mathrm{p}=0.0206)$, the same occurred for comparisons with chlorhexidine $(\mathrm{p}<0.0001)$. It can be concluded that mastic and pomegranate tinctures were toxic against Artemia salina in the different concentrations tested.

Keywords: Punicaceae; Bursera; Toxicity Tests.

\begin{abstract}
Resumen
Este trabajo tuvo como objetivo evaluar la toxicidad de las tinturas de masilla (Myracrodruon urundeuva) y granada (Punica granatum) a través del bioensayo con Artemia salina. Se agregaron diferentes concentraciones de colorante $(25,50,75,150,250,500,750$ y $1000 \mu \mathrm{g} / \mathrm{mL})$ a los nauplios y se evaluó la concentración letal promedio (CL50) mediante análisis Probit utilizando el software StatPlus ${ }^{\circledR}$ (AnalystSoft, EE. UU.), obteniendo los intervalos de confianza superior e inferior, después de un período de exposición de 24 horas. Los extractos se consideraron activos cuando los valores eran $<1000 \mathrm{ug} / \mathrm{mL}$. Se realizaron curvas de supervivencia y pruebas de LogRank para todos los extractos a una concentración de $250 \mu \mathrm{g} / \mathrm{mL}$ contra control negativo (solución salina) y control positivo (clorhexidina), utilizando el software GraphPad® Prism (GraphPad Software, EE. UU.). Para la masilla, la CL50 fue de $607,87 \pm 38,73$, para la granada, 464,98 $\pm 28,72$, considerándose los dos tinturas activas. En comparación con la solución salina, hubo una diferencia significativa en las curvas de supervivencia $(p=0,0206)$, lo mismo ocurrió para las comparaciones con clorhexidina $(\mathrm{p}<0,0001)$. Se puede concluir que las tinturas de masilla y granada resultaron tóxicas contra Artemia salina en las diferentes concentraciones ensayadas.
\end{abstract}

Palabras clave: Punicaceae; Bursera; Pruebas de Toxicidad.

\title{
1. Introdução
}

A fitoterapia baseada em compostos derivados de plantas é uma estratégia medicinal alternativa para o alívio dos sintomas e a cura de doenças. As folhas de Myracrodruon urundeuva, uma planta medicinal também conhecida como "aroeira", têm sido utilizadas na medicina tradicional como curativas, anti-úlceras e anti-inflamatórias no tratamento de doenças esqueléticas no Brasil, mas seu papel na toxicidade das células ósseas e na formação óssea, continua a ser estabelecido (Matos et al. 2019)

A aroeira é uma espécie medicinal brasileira, comum na região semiárida do nordeste brasileiro, cuja casca de caule é amplamente utilizada na medicina popular. É uma espécie em extinção, apresentando como principais componentes bioativos taninos e chalconas (Calou et al. 2014). Machado \& Oliveira (2014) relataram que a aroeira-do-sertão é uma planta utilizada na medicina tradicional nordestina e em alguns países da América do Sul, como fitoterápico na Odontologia, onde os extratos dela são obtidos através do preparo das folhas, raízes, entrecasca e casca do tronco e galhos. Dentre do os efeitos da aroeira estão a ação antimicrobiana, anti-inflamatória e cicatrizante. Além disso, apresenta atividade antifúngica equivalente ao controle frente à algumas cepas de Candida avaliadas (Freires et al. 2011). Estes mesmo autores, em 2018, fizeram uma revisão sistemática para identificar quais agentes naturais isolados têm efeitos na prevenção da perda óssea em um modelo de periodontite induzida por ligadura. $\mathrm{O}$ gel do extrato de aroeira mostrou forte eficácia protetora óssea alveolar na periodontite induzida em ratos. No entanto, observaram que pesquisas adicionais devem preencher a lacuna entre os resultados do estudo em ratos e a eficácia clínica e a toxicidade a longo prazo em humanos. Sugeriram a realização de outros estudos microbiológicos, toxicológicos e clínicos para verificar a viabilidade de uso na Odontologia.

A planta Punica granatum, também conhecida como romã, é indicada na prática odontológica, pois apresenta atividade antisséptica, antiviral e adstringente; além disso, possui efeito sobre um grande número de bactérias e, quando utilizada com finalidade antisséptica na fórmula de enxaguatório contendo $10 \%$ do extrato fluido da planta, promove significativa redução da recolonização bacteriana (Cachoeira et al. 2014). No entanto já foi demonstrado que a formulação do dentifrício com extrato de romã mostra eficiência na primeira semana de uso diminuindo significativamente o índice de biofilme, porém, não apresenta redução do número de estreptococos do grupo mutans (Argenta et al. 2012). Tavangar et al. (2019) observaram que o gel de romã tem um efeito bem-sucedido no controle e tratamento da estomatite aftosa recorrente, 
podendo ser considerado como um tratamento acessível e barato. Da mesma maneira foi demonstrado que os extratos metanólico e etanólico da casca e raiz da romã apresentam atividades anti-cândida e, portanto, eles podem ser considerados como enxaguatório bucal ou creme dental para prevenir e tratar infecções por Candida albicans na cavidade oral (Lavaee et al. 2018).

Apesar do efeito desejado ser obtido em alguns estudos com estas plantas medicinais, necessita-se de estudos para comprovar o seu mecanismo de ação e definir condições seguras para uso em patologias específicas. As plantas medicinais não estão livres de efeitos ou reações indesejáveis, pois precisam ser consumidas com cautela e seguindo o que preconiza a literatura. $\mathrm{O}$ uso racional das drogas vegetais é uma ação que precisa ser mais bem esclarecida para a população, além de necessitarem de mais e melhores estudos que comprovem suas características farmacológicas ou não, bem como seus possíveis males à saúde dos usuários, visto que determinadas plantas medicinais largamente utilizadas pela população, já apresentaram, em algumas concentrações, efeitos tóxicos, citotóxicos, genotóxicos e mutagênicos (Bezerra et al. 2016).

Toxicidade é uma particularidade referente a substância química que pode apresentar um potencial de produzir danos aos organismos vivos. Pode estar relacionada com o tempo de exposição e concentração da substância. (Rodriguez et al. 2009). Os efeitos da toxicidade de uma substância sobre organismos podem ser de natureza aguda ou crônica. Os efeitos agudos apresentam resultados súbitos e ligeiros em indivíduos apresentados a um estímulo; os mais frequentemente encontrados são letalidade e imobilidade (Massaro, 2006). A finalidade dos testes de toxicidade aguda é indicar a concentração de uma substância que pode acarretar danos em um conjunto de indivíduos que estão sendo avaliados. A exposição dos indivíduos à substância testada em um intervalo de tempo variável entre 24 horas e 96 horas. Os métodos de bioensaios são opções exequíveis, de curta duração e acessíveis (Rodriguez et al. 2009).

O uso de animais aquáticos para ensaios de toxicidade é frequente, dentre eles o microcrustáceo de água salgada Artemia salina. A espécie é comumente utilizada devido à simplicidade de manuseio e baixo custo, favorecendo seu uso em diversos estudos, pois é possível determinar por ensaios de toxicidade aguda, a Concentração Letal para 50\% da população teste (CL50) dos compostos bioativos em extratos de plantas (Alves et al. 2000) e a toxicidade para este crustáceo tem demonstrado uma boa correlação com a atividade citotóxica contra tumores humanos (Mclaughlin et al. 1991), sendo justificado o seu uso em bioensaios.

Diante do exposto, o presente estudo teve por objetivo determinar o potencial toxicológico das tinturas de aroeira e de romã, para nortear futuros estudos e verificar se tais espécies, amplamente utilizadas na medicina popular, apresentam atividade tóxica, por meio do bioensaio da Artemia salina.

\section{Metodologia}

O presente estudo trata-se de uma pesquisa laboratorial, de natureza quantitativa (Pereira et al. 2018). A toxicidade das tinturas foi testada no modelo da Artemia salina baseada na técnica descrita por Meyer et al. (1982). As tinturas de aroeira e de romã a $10 \%$ foram obtidas em farmácia de manipulação local. As soluções foram homogeneizadas e para o volume final foram adicionados $5 \mathrm{~mL}$ de solução salina de sal marinho, obtendo-se concentrações de 25, 50, 100, 150, 250, 500, 750 e 1000 $\mu \mathrm{g} / \mathrm{ml}$.

\subsection{Preparo das Artemias salinas}

Para a eclosão dos cistos de Artemia salina realizou se um preparo da água do mar artificial, no qual foi utilizado sal marinho (30 g), água destilada (1,5 1) e o pH foi controlado para 8 a 9, com bicarbonato de sódio. Em um aquário foram incubados os cistos $(3 \mathrm{~g})$, mantidos sob temperatura de $28^{\circ} \mathrm{C}$, com um termostato, sob aeração constante e iluminação artificial (20 w), durante um período entre 24 e 30 horas. 


\subsection{Contagem dos náuplios}

Após a eclosão, foram transferidos 10 náuplios para cada tubo de ensaio contendo solução salina (controle negativo), dicluconato de clorexidina $0,12 \%$ (controle positivo) e as tinturas nas diferentes concentrações $(25,50,100,150,250,500,750$ e $1000 \mu \mathrm{g} / \mathrm{mL}$ ). Após 24 e $48 \mathrm{~h}$ contaram-se os microcrustáceos vivos e mortos (foram considerados vivos os organismos que apresentaram movimento quando observados próximos à fonte luminosa durante dez segundos). O teste foi realizado em triplicata.

\subsection{Análise Estatística}

Para obtenção dos valores da concentração letal para matar 50\% dos náuplios (CL50), foi utilizada a análise de Probit através do software StatPlus ${ }^{\circledR}$ (AnalystSoft, USA), obtendo-se os intervalos de confiança superior e inferior, após um período de exposição de 24 horas. As tinturas avaliadas foram consideradas ativas quando, no ensaio de toxicidade sobre a Artemia salina, os valores foram $<1000 \mu \mathrm{g} / \mathrm{mL}$. Curvas de sobrevivência e testes de LogRank foram realizados para todos os extratos na concentração de $250 \mu \mathrm{g} / \mathrm{mL}$ contra controle negativo (Salina) e controle positivo (Clorexidina), utilizando o software GraphPad® Prism (GraphPad Software, USA).

\section{Resultados}

Os dados calculados pela Análise Probit para todas as preparações das plantas medicinais estão apresentados na Tabela 1 e indicam a CL50 \pm erro-padrão e os respectivos intervalos de confiança obtidos para o limite superior e inferior, com nível de intervalo de confiança $=1 \%$.

Tabela 1. Dados de toxicologia obtidos para as plantas medicinais no bioensaio de Artemia salina.

\begin{tabular}{lcccc}
\hline \multicolumn{1}{c}{ Planta Medicinal } & $\begin{array}{c}\text { CL50 } \\
(\boldsymbol{\mu g} / \mathbf{m L})\end{array}$ & Erro-padrão & $\begin{array}{c}\text { Limite inferior } \\
(\boldsymbol{\mu g} / \mathbf{m L})\end{array}$ & $\begin{array}{c}\text { Limite superior } \\
(\boldsymbol{\mu g} / \mathbf{m L})\end{array}$ \\
\hline Aroeira & 607,87 & 38,73 & 507,04 & 708,71 \\
Romã & 464,98 & 28,52 & 390,55 & 539,41
\end{tabular}

CL50 - Concentração Letal para 50\% da população teste, $\mu \mathrm{g} / \mathrm{mL}$ - microgramas por mililitro. Fonte: Autores.

Para a aroeira a CL50 foi de 607,87 $\pm 38,73$, já para a romã, de 464,98 $\pm 28,72$, sendo ambas as tinturas consideradas ativas. A viabilidade dos náuplios de Artemia salina frente as diferentes plantas medicinais nas concentrações de $250 \mu \mathrm{g} / \mathrm{mL}$ foram avaliadas experimentalmente, indicando que as curvas de sobrevivência foram estatisticamente diferentes $(\mathrm{p}<0.0001)$, segundo o teste de LogRank.

As curvas de sobrevivência dos náuplios no bioensaio testadas contra amostras de Aroeira e Romã estão representadas nas Figuras 1 e 2, respectivamente, contra os grupos controle negativo (solução salina) e positivo (digluconato de clorexidina $0,12 \%)$, acompanhados dos valores calculados na análise estatística para o teste de LogRank durante 24h. 
Figura 1. Porcentagem de sobrevivência dos náuplios de Artemia salina (aroeira versus salina, p =0.0206, e aroeira versus clorexidina, $\mathrm{p}<0.0001$, segundo o teste de Logrank).
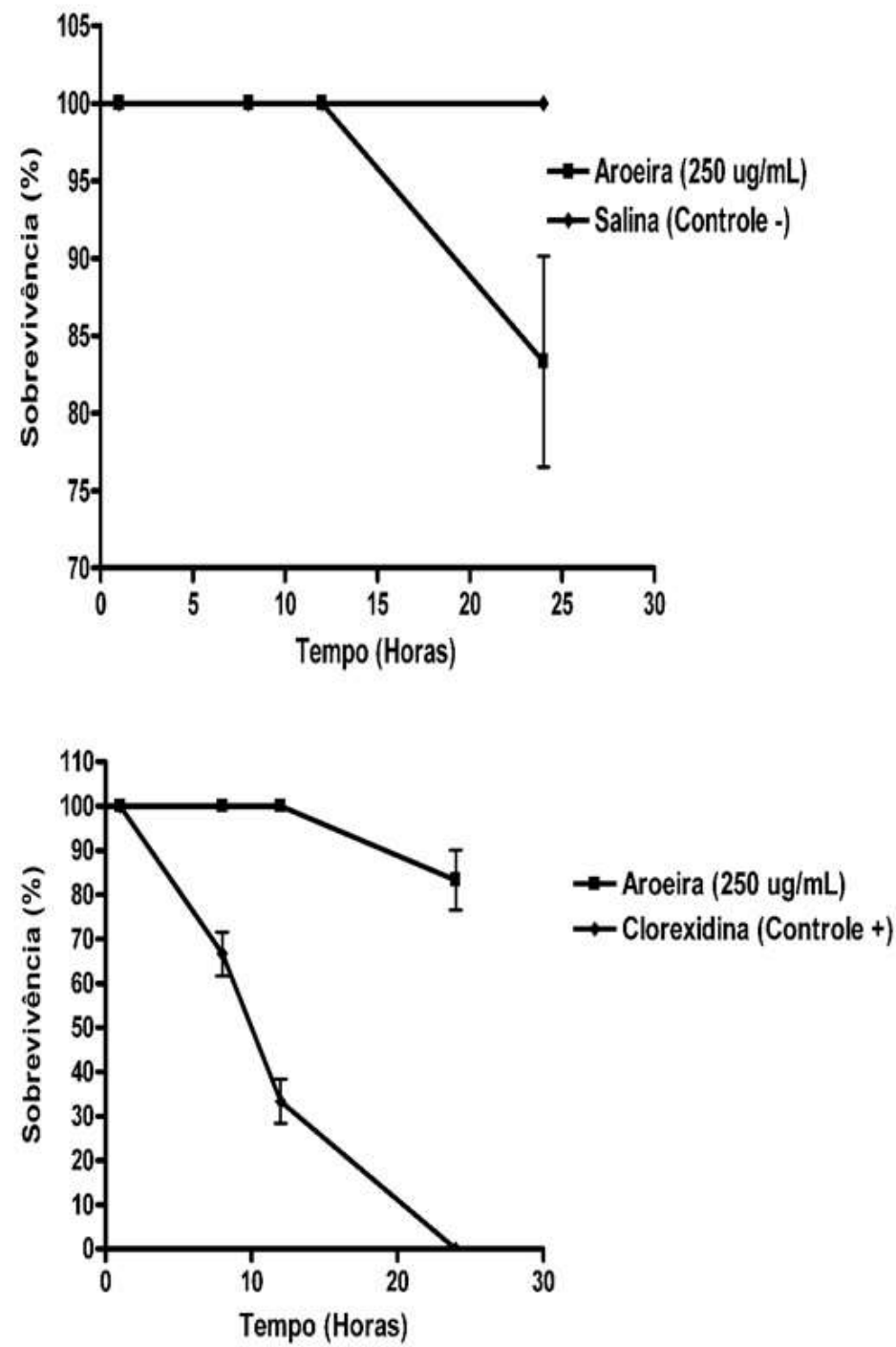

Fonte: Autores.

Quando comparada à solução salina, houve diferença significativa na curva de sobrevivência para a aroeira ( $\mathrm{p}=$ 0,00206), mostrando que a aroeira é tóxica quando comparada à solução salina. O resultado obtido para a solução salina foi o esperado, visto que é o meio em que os náuplios vivem. Quando comparada com a clorexidina, houve diferença estatisticamente significante ( $\mathrm{p}<0,0001)$, mostrando que a clorexidina é mais tóxica do que a aroeira. 
Figura 2. Porcentagem de sobrevivência dos náuplios de Artemia salina (romã versus salina, p =0.0206, e romã versus clorexidina, $\mathrm{p}<0.0001$, segundo o teste de Logrank).
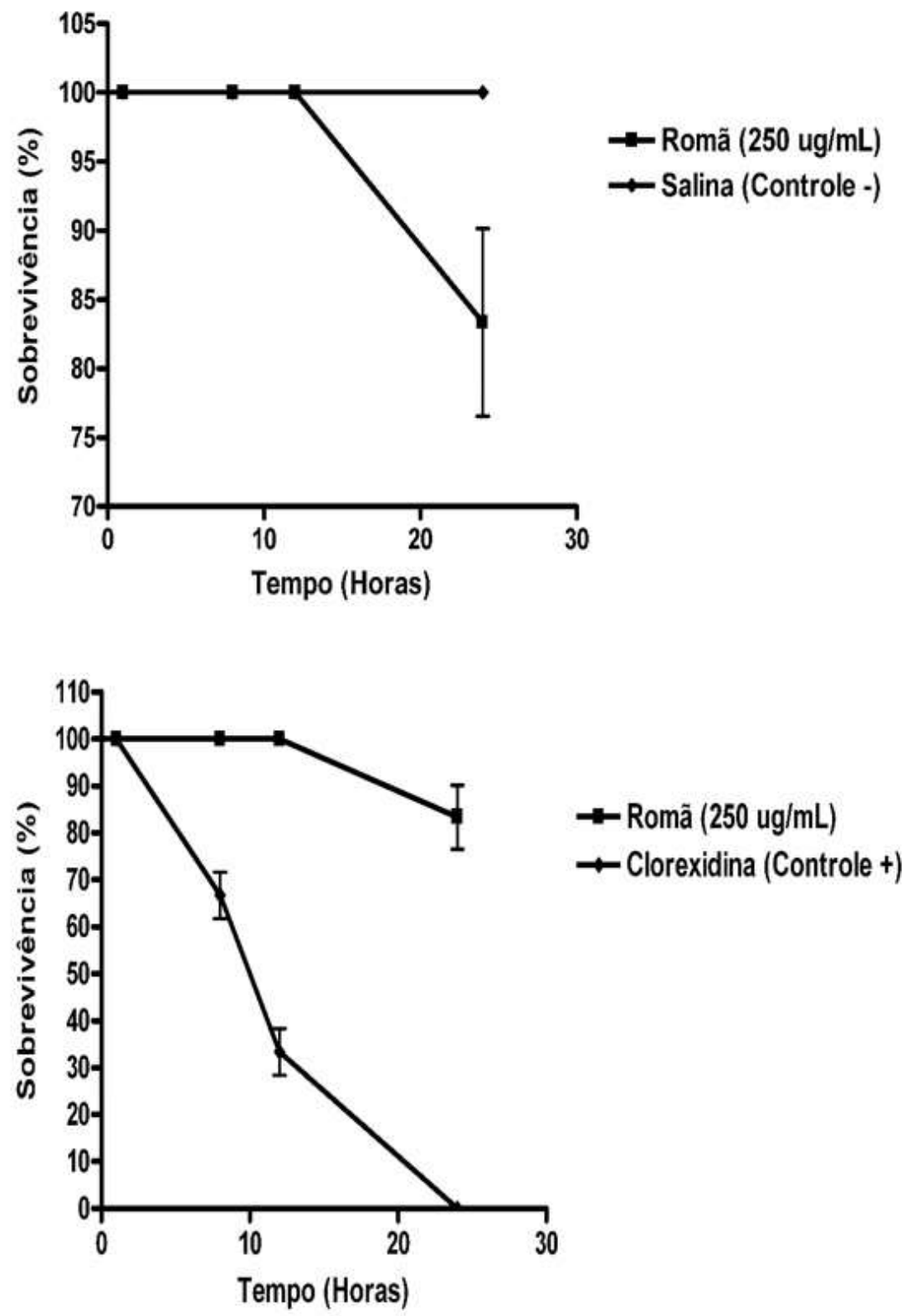

Fonte: Autores.

Quando comparada à solução salina, houve diferença significativa na curva de sobrevivência para a romã ( $\mathrm{p}=$ 0,00206), mostrando que a romã é tóxica quando comparada à solução salina. O resultado obtido para a solução salina foi o esperado, visto que é o meio em que os náuplios vivem. Quando comparada com a clorexidina, houve diferença estatisticamente significante ( $\mathrm{p}<0,0001)$, mostrando que a clorexidina é mais tóxica do que a romã. 
Figura 3. Porcentagem de sobrevivência dos nauplios de Artemia salina frente a amostras de aroeira versus romã (p >0.05, segundo o teste de Logrank).

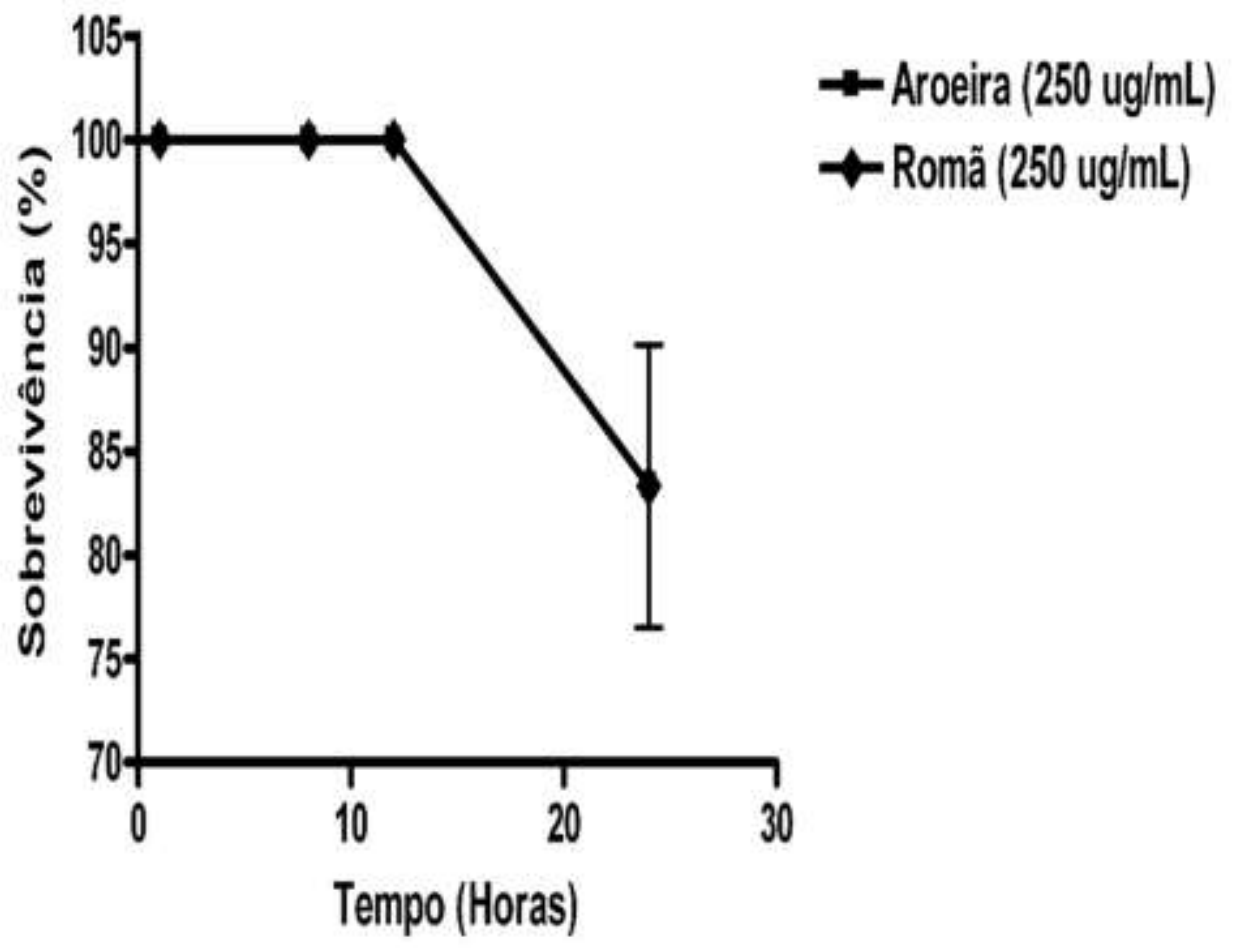

Fonte: Autores.

A Figura 3 demonstra que não ocorreu diferença estatisticamente significante entre as amostras de aroeira e romã no bioensaio de sobrevivência dos náuplios $(\mathrm{p}>0,05)$, onde ambas apresentaram certo grau de toxicidade.

\section{Discussão}

As plantas medicinais vêm sendo mundialmente empregadas na medicina popular. $\mathrm{O}$ uso indiscriminado de plantas sem qualquer conhecimento fitoquímico, farmacológico e principalmente toxicológico é de grande preocupação para a saúde. Assim, encontrar uma concentração segura e não letal para o uso terapêutico constitui-se uma etapa de grande importância.

O teste de toxicidade sobre a Artemia salina é um ensaio biológico amplamente utilizado devido à sua rapidez, confiança e baixo custo. A relação entre o grau de toxicidade e a concentração letal média (CL50) apresentada por extratos de plantas sobre larvas de Artemia salina, tanto extratos orgânicos, quanto extratos aquosos com valores de CL50 acima de 1000 $\mu \mathrm{g} / \mathrm{mL}$, estes, são considerados atóxicos, baixa toxicidade quando a CL50 for superior a 500 $\mu \mathrm{g} / \mathrm{mL}$; moderada para CL50 entre 100 a $500 \mu \mathrm{g} / \mathrm{mL}$ e muito tóxico quando a CL50 foi inferior $100 \mu \mathrm{g} / \mathrm{mL}$ (Amarante, 2011). No presente estudo a tintura de aroeira foi considerada com baixa toxicidade e a tintura de romã, com toxicidade moderada, mas se faz necessária a realização de outros estudos para afirmar com segurança tal resultado.

A primeira publicação a utilizar o teste de Artemia salina é datada de 1956 (Michael et al. 1956), desde então, numerosos artigos utilizam essa abordagem para a avaliação de limites toxicológicos de diversas substâncias, principalmente extratos de plantas. Os estudos de determinação da CL50 em Artemia salina mostram forte correlação com valores encontrados em camundongos Swiss, o que mostra que esse bioensaio proporciona grandes vantagens na avaliação de toxicidade de 
derivados de plantas, pois proporcionam informações úteis no prosseguimento de estudos toxicológicos mais avançados, então podendo ser utilizado como ensaio preliminar de químicos tóxicos em mamíferos para determinar a janela de ação tóxica (Arcanjo et al. 2012, Migliore et al. 1997, Nunes et al. 2006).

Diversas plantas têm sido estudadas com relação à toxicidade, por meio da utilização do extrato bruto e óleo essencial, visto que são substâncias com propriedades farmacológicas (Venturoso et al. 2011). O estudo de Hyacienth \& Almeida (2015) que avaliou a toxicidade da Lamuci (Pseudoxandra cuspidata Maas) contra Artemia salina encontrou uma toxicidade média de 605,92 , resultado semelhante ao que foi encontrado para a aroeira no presente estudo. Esta toxicidade pode ser justificada pela presença de alcaloides e/ou compostos fenólicos na planta (Hyacienth \& Almeida, 2015). A espécie de Caesalpinia férrea (Pau ferro) também demonstrou baixa toxicidade frente à Artemia salina, com valor de CL50 de 822,63 (Lima et al. 2019). Resultados diferentes foram obtidos para as seguintes espécies: Bromelia laciniosa (Mart.), Ex schult (Macambira), Caesalpinia pyramidalis Tul (Catingueira), Cereus jamacaru DC (Mandacaru) e Nopalea cochenillifera L.Salm-Dyck (Palma), que foram consideradas atóxicas (CL50 > 1000) (Lima et al. 2019).

Em se tratando das mesmas plantas utilizadas no presente estudo, Almeida et al. (2009) avaliaram a toxicidade do extrato hidroalcóolico de aroeira, administrados por via intraperitoneal, em camundongos Swiss e encontrou alta toxicidade, o que corrobora com os resultados desta pesquisa. O estudo de Werkman (2009) avaliou a toxicidade da romã por meio de cultura celular de linhagens de fibroblastos humanos de mucosa oral e células de carcinoma epidermóide oral humano, e encontrou toxicidade em ambas as linhagens, concordando com os resultados deste estudo.

O presente estudo trata-se de um bioensaio, considerado essencial e preliminar no estudo de compostos com potencial atividade biológica, realizado em ambiente que permite o controle de variáveis, reduzindo o grau de subjetividade na aferição dos dados. Deve-se interpretar os resultados com cautela, para não ter a expectativa de obter o mesmo resultado para seres humanos, uma vez que se trata de experimentos realizado em outras espécies que não a humana.

\section{Considerações Finais}

As tinturas de aroeira e de romã foram tóxicas contra a Artemia salina nas diferentes concentrações testadas, porém a toxicidade foi considerada leve e moderada, respectivamente.

Mais estudos em animais e estudos clínicos em humanos, que testem a toxicidade das tinturas de aroeira e de romã, são necessários para comprovar tal resultado.

\section{Referências}

Almeida, A. C., Sobrinho, E. M., De Pinho, L., Souza, P. N. S., Martins, E. R., Duarte, E. R., Santos, H. O., Brandi, I. V., Cangussu, A. S. \& Costa, J. P. R. (2010) Toxicidade aguda dos extratos hidroalcoólicos das folhas de alecrim-pimenta, aroeira e barbatimão e do farelo da casca de pequi administrados por via intraperitoneal. Ciência Rural 40(1), 1-4.

Alves, T. M. A., Silva, A. F., brandão, M., Grandi, T. S. M., Smânia, E. F. A., Smânia Jr., A. \& Zani, C. L. (2000) Biological screening of Brazilian medicinal plants. Memórias do Instituto Oswaldo Cruz 95, 367-373.

Amarante, C. B., Müller, A. H., Póvoa, M. M. \& Dolabela, M. F. (2011) Estudo fitoquímico biomonitorado pelos ensaios de toxicidade frente à Artemia salina e de atividade antiplasmódica do caule de aninga (Montrichardia linifera). Acta Amazônica 41(3), 431-434.

Arcanjo, D. D., Albuquerque, A. C., Melo-Neto B., Santana, L. C., Medeiros, M. G. \& Citó, A. M. (2012) Bioactivity evaluation against Artemia salina Leach of medicinal plants used in Brazilian Northeastern folk medicine. Brazilian Journal of Biology 72(1), 505-509.

Argenta, J. A., Pasqual, M., Pereira, C. V., Dias, D. R., Barbosa, R. A. \& Pereira, L. J. (2012) Efeito do extrato de romã (Punica granatum) sobre bactérias cariogênicas: estudo in vitro e in vivo. Arquivos em Odontologia 48(4), 218-226.

Bezerra, C. M., Oliveira, M. A. S. (2016) Avaliação da toxicidade, citotoxicidade e genotoxicidade do infuso de malva-santa Plectranthus barbatus (Lamiaceae) sobre o ciclo celular de Allium cepa. Eletronic Journal of Pharmacy 13(3), 220-228.

Cachoeira, S. F., Dallanora, F., Dallanora, L. M. F. \& Masson, P. (2014) Uso da punica granatum em odontologia: desenvolvimento de enxaguatório bucal com extrato fluido de punica granatum. Ação Odonto 2(3), 44. 
Calou, I., Bandeira, M. A., Aguiar-Galvão, W., Cerqueira, G., Siqueira, R., Neves, K. R., Brito, G. A. \& Viana, G. (2014) Neuroprotective Properties of a Standardized Extract from Myracrodruon urundeuva Fr. All. (Aroeira-Do-Sertão), as Evaluated by a Parkinson's Disease Model in Rats. Parkinsons Disease 2014,519615

Freires, I. A., Alves, L. A., Jovito, V. C. \& Castro, R. D. (2011) Atividade antifúngica de Schinus terebinthifolius (Aroeira) sobre cepas do gênero Candida. Revista Odontológica do Brasil-Central 20(52), 41-45.

Freires, I. A., Santaella, G. M., Sardi, J. C. O. \& Rosalen, P. L. (2018) The alveolar bone protective effects of natural products: A systematic review. Archives of Oral Biology 87,196-203.

Hyacienth, D. C. \& Almeida, S. S. M. S. (2015) Estudo fitoquímico, toxicidade em Artemia salina Leach e atividade antibacteriana de Pseudoxandra cuspidata Maas. Biota Amazônia 5(4), 4-7.

Lavaee, F., Motaghi, D., Jassbi, A. R., Jafarian, H., Ghasemi, F. \& Badiee, P. (2018) Antifungal effect of the bark and root extracts of Punica granatum on oral Candida isolates. Current Medical Mycology 4(4), 20-24.

Lima, M. F. F., Silva, J. W. S. A., Silva, J. K., Moura, A. H. N., Lopes, L. R. F., Cordeiro, B. A., Cordeiro, R. P. \& Melo, A. F. M. (2019) Avaliação toxicológica através do bioensaio com Artemia salina Leach de espécimes vegetais pertencentes à caatinga. Brazilian Journal of Health Research 2(6), 59505963.

Machado, A.C. \& Oliveira, R.C. (2014) Medicamentos Fitoterápicos na odontologia: evidências e perspectivas sobre o uso da aroeira-do-sertão (Myracrodruon urundeuva Allemão). Revista Brasileira de Plantas Medicinais 16(2), 283-289.

Massaro, F. C. Estudos ecotoxicológicos com Hydra viridissima (Cnidaria: Hydrozoa), Dissertação, Escola de Engenharia de São Carlos, Universidade de São Paulo, São Carlos SP 2006.

Matos, A. A., Oliveira, F. A., Machado, A. C., Saldanha, L. L., Tokuhara, C. K., Souza, L. P., Vilegas, W., Dionísio, T. J., Santos, C., Peres-Buzalaf, C., Dokkedal, A. L. \& Oliveira, R. (2019) An extract from Myracrodruon urundeuva inhibits matrix mineralization in human osteoblasts. Journal of Ethnopharmacology 237, 192-201.

Mclaughlin, J. L., Chang, C. J. \& Smith, D. L. (1991) "Bench-top" bioassays for the discovery of bioactive natural products. Studies in Natural Product Chemistry 9,383-409.

Meyer, B. N., Ferrigni, N. R., Putnam, L. B., Jacobsen, L. B., \& Nichols De Mclaughlin, J. L. (1982) Brine shrimp: a convenient general bioassay for active plant constituents. Journal of Medicinal Plants Research 45, 31-34.

Michael, A. S., Thompson, C. G. \& Abramovitz, M. (1956) Artemia salina as a Test Organism for Bioassay. Science 123(3194),464.

Migliore, L., Civitareale, C., Brambilla, G. \& Di-Delupis, G. D. (1997) Toxicity of several important agricultural antibiotics to Artemia. Water Research 31(7), 1801-1806.

Nunes, B.S., Carvalho, F.D., Guilhermino, L.M. \& Stappen, G.V. (2006) Use of the genus Artemia in ecotoxicity testing. Environmental Pollution 144(2),453456.

Pereira, A. S., Shitsuka, D. M., Parreira, F. J. \& Shitsuka, R. (2018) Metodologia da Pesquisa Científica. 1. ed. - Santa Maria, RS: UFSM, NTE, 119 p.

Rodriguez, A. G., Teixeira, O. M., Salles, F. G., Vital, J. P. \& Peixoto, D. S. (2009) Bioensaio com artemia salina para detecção de toxinas em alimentos vegetais. Estudos Goiânia 36(5/6), 795-808.

Tavangar, A., Aslani, A. \& Nikbakht, N. (2019) Comparative Study of Punica granatum Gel and Triadent Oral Paste Effect on Recurrent Aphthous Stomatitis, a Double-Blind Clinical Trial. Journal of Dentistry (Shiraz) 20(3),184-189. 